\title{
Measuring Disease Progression in Osteoarthritis
}

\author{
Laura L. Laslett, BSc (Hons), MMedSci, GDPH, PhD ${ }^{1}$ \\ Jean-Pierre Pelletier, $M D^{2}$ \\ Flavia M. Cicuttini, MBBS, PhD ${ }^{3}$ \\ Graeme Jones, MBBS(Hons), MMedSCi, MD ${ }^{1}$ \\ Johanne Martel-Pelletier, $P h D^{2, *}$
}

\author{
Address \\ ${ }^{1}$ Menzies Institute for Medical Research, University of Tasmania, Private Bag 23, \\ Hobart, TAS, 7000, Australia \\ ${ }^{*}, 20$ steoarthritis Research Unit, University of Montreal Hospital Research Centre \\ (CRCHUM), 900 rue Saint-Denis, Suite R11.412, Montreal, QC, H2X 0A9, Canada \\ Email: jm@martelpelletier.ca \\ ${ }^{3}$ Department of Epidemiology and Preventive Medicine, School of Public Health \\ and Preventive Medicine, Monash University, Alfred Hospital, Prahran 3181, \\ Melbourne, Australia
}

Published online: 7 April 2016

(C) Springer International Publishing AG 2016

This article is part of the Topical Collection on Osteoarthritis

Keywords Osteoarthritis - Knee osteoarthritis - Hand osteoarthritis - Magnetic resonance imaging - Radiographs . Ultrasound

\section{Opinion statement}

Radiographs are a commonly used tool to assess disease progression in osteoarthritis $(0 \mathrm{~A})$. However, it is not the preferred method of defining and following $0 \mathrm{~A}$ progression. While it is moderately responsive to change in terms of standardised response means (SRM), it is insensitive to change in cartilage measures. MRI offers a much better assessment, and $\mathrm{OA}$ features are much better targeted for defining and following the disease progression. Using MRI, cartilage volume/thickness loss predicts knee replacement and has similar levels of sensitivity to discriminate treatments in clinical trials. Cartilage defect is another target which was found to also be an independent predictor of some OA outcomes. In addition to cartilage, another MRI target for diagnosis and assessment of $\mathrm{OA}$ progression is subchondral bone alterations, especially bone marrow lesions (BMLS). BMLs independently predict OA outcomes including knee replacement and are sensitive to progression. Targeting of BMLs by bone remodelling agents demonstrates that the natural history of the disease could be diminished. MRI-detected effusion and synovitis are also promising targets of $\mathrm{OA}$ progression. In addition, alterations in these tissues are promising outcome measures for clinical trials. Newer MRI methods of cartilage quality assessment, including signal intensity on T1 images and dGEMRIC, have been proposed for assessing early cartilage loss. However, the requirement for a contrast agent with dGEMRIC precludes its widespread use on safety grounds. Another promising imaging 
modality for $\mathrm{OA}$ is ultrasound. However, this approach warrants further exploration as, although it has demonstrated a wide set of advantages over other imaging modalities, there are still major limitations. Evidence for use of ultrasound indices for assessing disease progression is limited. At present, this imaging modality may be better suited as a diagnostic and an explanatory tool within clinical practice rather than large-scale studies or clinical trials.

\section{Introduction}

Osteoarthritis (OA) is currently defined as a 'progressive disease of synovial joints, representing failed repair of joint damage that results from stresses initiated by an abnormality in any of the synovial joint tissues... this ultimately results in the breakdown of cartilage and bone, leading to symptoms of pain, stiffness and functional disability' [1]. Such a concept of OA as being only a degenerative disease or 'wear and tear' is now considered outdated $[2,3]$. OA is a complex disease involving all the tissues of the joint as well as inflammation. Emerging research suggests that $\mathrm{OA}$ is a collection of heterogeneous pathologies that result in a common outcome (pain and joint destruction), rather than being one homogeneous disease. This heterogeneity includes different subtypes and structures affected, where OA processes begin, and risk factors, all depending on the joints affected.

In addition to cartilage degeneration, specific phenotypes/subtypes of OA proposed in the literature include a bone-specific subtype [4], predominantly bone marrow lesions in the subchondral bone [5], traumatic (acute or repetitive) which may be initiated by lesions at the anterior cruciate ligament following mechanical disruption [6-8], or as a result of knee meniscectomy, metabolic (including systemic inflammatory), ageing, genetic and pain [9], as well as atrophic (few osteophytes with severe joint space narrowing (JSN)), hypertrophic (large osteophytes, little JSN) $[10,11]$ ) and traumatic (acute or repetitive). These also vary in the main joint site(s) affected. Hip and knee OA can result from the majority of the subtypes, and post-traumatic stresses are proposed to result in less common types of OA including the thumb, shoulder, elbow and ankle OA [9].

The majority of studies assessing measurement of OA progression have been done in the knees, first using radiographs, and more recently employing other tools including MRI and ultrasound, but the latter to a much lesser extent and targeting specific and fewer joint structures than MRI.

\section{Radiographs}

\section{Radiographic definitions of osteoarthritis}

Historically, radiography has been the most common imaging modality to assess $\mathrm{OA}$. The most commonly used radiographic definitions of OA are the Kellgren and Lawrence (KL) classification [12], which grades the joint as a whole (grade 0 , no OA; to grade 4, severe), based on marginal osteophytes, JSN, subchondral bone sclerosis and altered shape of the bone.

The more recent OARSI system [13] grades individual features of OA (JSN, subchondral lucency, marginal osteophytes, subchondral sclerosis) on a 0-3 scale (where 0 , absent and 3, severe), with no grading for the joint overall.

Although assessment of joint structural features on radiographs remains widely used for diagnosis and for staging OA, this technology is two dimensional, subject to measurement error due to joint positioning issues, typically used in a semi-quantitative manner and does not allow visualisation of noncalcified tissues [14], as well as being a poor predictor of clinical outcomes [15] and only weakly associated with symptoms [16-19]. 
Radiographic assessment of knee joints has historically been used as a proxy measure of cartilage volume, due to favourable associations between joint space width (JSW) and cartilage volume cross-sectionally. However, JSN is not sensitive for cartilage loss since cartilage loss frequently occurs without a corresponding progression of JSN. Correlations between changes in JSW and cartilage volume in OA cohorts were observed in some studies, but were weak over long periods of observation [20-22], while others reported no correlation over 2 years [23, 24]. In addition, this method also shows unexplained variability, which could reflect change in meniscal position or structure alteration as well as cartilage morphology $[22,25-28]$. Therefore, change in JSW is a poor proxy for change in cartilage volume longitudinally. Moreover, although this method can be assessed in a quantitative manner (mJSW), the assessment of JSN in both the KL and OARSI classifications is only semi-quantitative (range $0-3$ or $0-4$, respectively).

Overall, although change in grade of radiographic assessment of OA knee joints remains the "gold standard" for assessing disease modification in clinical trials [29] and is mandated by the USA Food and Drug Administration and European Medications Agency as a proxy endpoint to determine efficacy of disease modifying OA drugs (DMOAD), this method is very insensitive to cartilage change.

Measuring progression of $0 \mathrm{~A}$ using radiographs

Measurement of knee JSW obtained from radiographs in persons with knee OA is moderately responsive to change (standardised response mean (SRM) of 0.33, increasing to 0.57 in studies longer than 2 years duration); however, the interreader coefficient is high (ICC of 0.97) [30]. These estimates are inferior to comparable MRI measures of cartilage [21, 24, 31, 32]. Radiographic grade (as assessed by KL grade) does predict risk of knee replacements over 3-8-year periods $[33,34]$, which is unsurprising, given that decisions for joint replacement are based on radiographs. However, this association is not independent of cartilage loss in some cohorts [35], and is only one of the predictors, for example for pain [33, 36].

In hand OA, site-specific scoring systems have been developed for the assessment of radiographic progression, such as the Verbruggen-Veys anatomical phase score [37], and the quantitative Ghent University score system (GUSS) used in erosive hand OA [38]. Several reviews have investigated the reproducibility and sensitivity to change of the numerous hand scoring methods using radiographic progression [39-41], and data showed that the estimated SRM values for four of them were modest (0.24 to 0.29) [40] and all semi-quantitative methods were comparable [39, 40]. However, with radiographic methods, the time required to demonstrate a change in hand OA is less than for knee OA, as it can detect differences in treatment in 6 months or less [42]. Quantitative JSW of the hand is a valid method to measure joint space and showed a good relation with clinical features in a case-control study [43], but this remains to be demonstrated in longitudinal studies.

\section{Magnetic resonance imaging}

Assessment of osteoarthritis

MRI allows assessment of the whole joint; it enables visualisation of cartilage and a large range of other structures, including those associated with pain. These 
can be assessed over a continuum from normal to diseased joint (OARSI grade 1 medial JSN, where more than $10 \%$ of cartilage is already lost [14]), through to end-stage OA (OARSI grade 3, KL grade 4).

\section{Diagnosis of OA using MRI}

Based on a systematic review of 25 MRI studies [44], tibiofemoral OA is currently defined as requiring a combination of features: either an osteophyte and full thickness cartilage loss or an osteophyte or full thickness cartilage loss, plus two or more of the following features: bone marrow lesion (BML) or cyst not associated with meniscal or ligamentous attachments, meniscal pathology, partial thickness cartilage loss (in the absence of full thickness cartilage loss), or bone attrition. Patellofemoral OA requires an osteophyte and partial or full thickness cartilage loss, all in the patella and/or anterior femur regions [44]. MRI-based scoring systems have also been proposed in hand OA [45], a refinement of the Oslo hand OA scoring system, including osteophytes, JSN, bone erosions/attrition, cysts, malalignment, synovitis and BML in the distal and proximal interphalangeal (PIP) joints.

The MRI-based definitions of OA include cartilage, but also other important structures that would be considered part of putative OA phenotypes, such as $\mathrm{BML}$, meniscal alterations, synovitis (synovial membrane thickness, effusion) and ligament pathology (Fig. 1) especially if they lead to functional disabilities [1]. All these structures could be the causal pathways of pathological processes of OA and are therefore potentially suitable as targets for therapy or as proxy endpoints for assessing disease progression.

MRI assessment of cartilage morphology is now recommended for the evaluation of disease progression as an endpoint for clinical trials [29, 46]. Tissues other than cartilage are also increasingly being used and include BMLs, meniscal alterations, synovitis and effusion. Associations between structural features of knee OA as seen on MRI and knee OA outcomes are summarised in Table 1.

Cartilage volume as assessed by MRI is a direct measure, and its level of loss has been shown to be better responsive to change than JSW [21, 24, 30, 32, 47]. Unsurprisingly, quantitative measures also have superior sensitivity to cartilage changes compared to semi-quantitative scoring [31, 48•].

Rate of cartilage loss is a good measure of progression as it predicts risk of knee replacements $[35,46,49]$. Every $1 \%$ increase in the rate of tibial cartilage loss over 2 years confers a $20 \%$ increased risk of knee replacement at 4 years [35]. Data from clinical trials in knee OA have shown that reduction in cartilage loss by potential DMOADs can reduce the cumulative incidence of total knee replacement over time $[49,50]$. Cartilage thickness has also been used to assess rates of disease progression, and data demonstrate that both the measurement of cartilage thickness and that of volume provide the same level of sensitivity to estimate cartilage loss in a clinical trial [51]. Loss of cartilage thickness was also found associated with increased risk of knee replacement [52].

It is now possible to accurately and feasibly measure change in cartilage volume over a 6-month period [53,54], although time frames of 24 months 

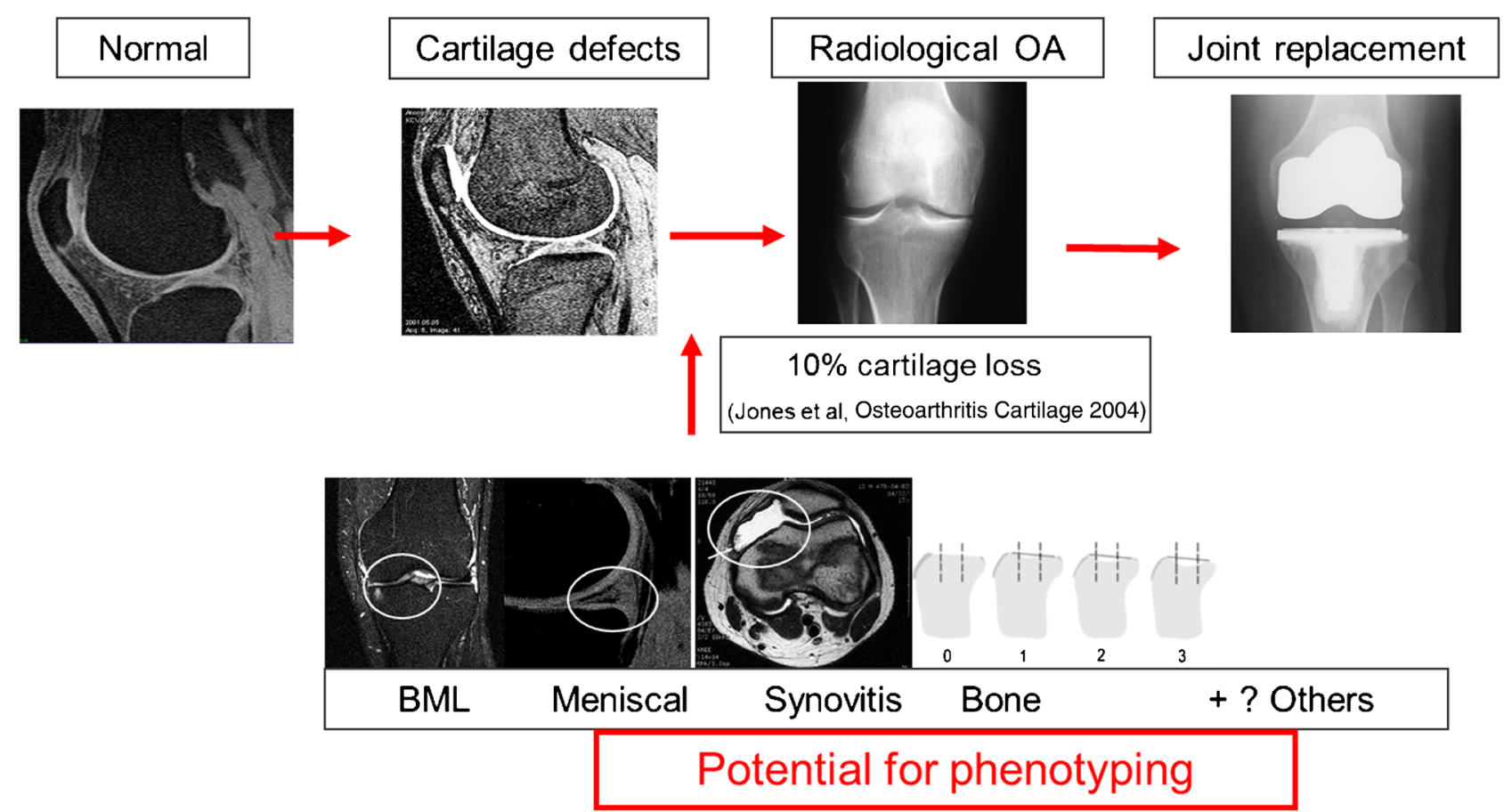

Fig. 1. Progression from a normal joint to a joint affected by end-stage osteoarthritis. Note: reference: [14].

and sample sizes of $\sim 200$ are usually used for clinical trials with cartilage endpoints; this is much shorter and involves fewer patients than required for knee radiographic endpoints.

\section{Cartilage assessment: other measures}

\section{Cartilage defects}

Defects in the cartilage are common in people with knee OA and have poor capacity for repair; although there is some evidence that reduction in weight is associated with reduction in defect severity, at least in a midlife cohort [55]. Such defects are associated with reduced cartilage volume and patellar cartilage volume cross-sectionally in people with symptomatic knee OA [56]. Longitudinally, change in cartilage defects was associated with tibiofemoral cartilage loss over 10 years in a midlife cohort [55], and greater number of cartilage defects predicted joint replacement over 4 years, which was not confounded by age, gender, BMI, symptoms, annual percentage cartilage loss or radiographic severity [56]. SRMs for cartilage defects were similar in magnitude to those obtained for cartilage volume [32].

A recent trend in OA research is the investigation of changes in cartilage using various MR imaging modalities. These should enable assessment of progression of cartilage loss in earlier stages of OA and even before loss of this tissue. The methods most tested are the T1 cartilage signal intensity, delayed gadolinium-enhanced MRI of cartilage (dGEMRIC), and T2 relaxation time mapping [57], all of which require specialised MRI sequences. Reduction in mean signal intensity of cartilage on T1-weighted MR images 


\begin{tabular}{|c|c|c|c|c|}
\hline & MRI structures/endpoints & $\begin{array}{l}\text { Progression } \\
\text { of knee ROA }\end{array}$ & $\begin{array}{l}\text { Knee joint } \\
\text { replacement }\end{array}$ & $\begin{array}{l}\text { Evidence for } \\
\text { modifiability }\end{array}$ \\
\hline Cartilage volume & - & & Yes & \\
\hline Cartilage thickness & & & Yes & \\
\hline Cartilage defects & $\begin{array}{l}\text { Yes (CV loss), change in } \mathrm{T} 2 \\
\text { relaxation time }\end{array}$ & & Yes & Yes \\
\hline \multicolumn{5}{|l|}{ Other cartilage measures: } \\
\hline $\begin{array}{l}\text { T1 cartilage signal } \\
\text { intensity }\end{array}$ & Yes: reduced cartilage thickness & & & \\
\hline dGEMRIC & $\begin{array}{l}\text { Yes: increased cartilage thickness } \\
\text { (swelling?) } \\
\text { No: cartilage thinning }\end{array}$ & & & Yes \\
\hline $\begin{array}{l}\text { Bone marrow lesions } \\
\text { (BMLs) }\end{array}$ & Yes: CV loss, progression of defects & Yes & Yes & Yes \\
\hline Synovitis & No & & Yes & \\
\hline Effusion-synovitis & $\begin{array}{l}\text { Yes (cartilage defects, BMLs, } \\
\text { CV loss) }\end{array}$ & & Yes & Yes \\
\hline Meniscal damage/tears & Yes (CV loss) & & Yes & \\
\hline $\begin{array}{l}\text { Infrapatellar fat pad } \\
\text { volume }\end{array}$ & Yes (CV loss, cartilage defects) & - & - & \\
\hline CV cartilage volume & & & & \\
\hline
\end{tabular}

is associated with reduced cartilage thickness over 2.9 years, suggesting that low cartilage signal intensity on T1 MRI may reflect early OA changes [58]. dGEMRIC is a measure of cartilage composition and is used to assess the relative distribution of glycosaminoglycans in cartilage, on the T1 sequences of MR images [59]. A low dGEMRIC index indicates normal cartilage, and a high dGEMRIC index indicates degraded cartilage. Reduced dGEMRIC values are associated with increased cartilage thickness in the medial tibiofemoral compartment [60], which may represent swelling of cartilage in the early stages of degeneration. Weight loss has been demonstrated to improve quality (increased proteoglycan content) and quantity (reduced cartilage thickness loss) of medial knee articular cartilage using dGEMRIC over 12 months [61], as has physical activity in young women over 3 months, as demonstrated by an improvement in T1(Gd) compared with the control group [62]. However, this technique uses a contrast agent, gadopentate dimeglumine $\left(\mathrm{Gd}-\mathrm{DTPA}^{2-}\right)$ and the USA Food and Drug Administration issued a report concerning the potentially serious side effects related to such contrast agent, particularly in older patients who constitute the great majority of OA patients, precluding its routine use or use in large clinical trials. The T2 relaxation time of articular cartilage is a function of the water content and collagen structure of the tissue. Measurement of the spatial distribution of the T2 relaxation time is associated with areas of increased or decreased water content, correlating with cartilage damage [57]. Higher baseline T2 is associated with greater 
cartilage thickness, higher cartilage volume [63] and fewer cartilage defects [64] cross-sectionally, and cartilage loss longitudinally [64]. However, longitudinal change in T2 relaxation time was inversely associated with grade of cartilage defects at baseline and follow-up, suggesting that once morphological cartilage defects occur, $\mathrm{T} 2$ values may be of limited use for evaluating further cartilage degradation [64].

BMLs predict site-specific progression of knee JSN in those with symptomatic knee OA (ORs 8.9 medially and 5.9 laterally, reducing to 2.8 and 5.6, respectively, following adjustment for alignment and other covariates) [65]. Moreover, a combination of cartilage damage and BMLs was associated with progression of JSN [66]. BMLs are strong and independent predictors of knee cartilage loss, especially site-specific cartilage loss [67, 68, 69••], and progression of knee cartilage lesions [24, 28, 67, 68, 70-72]. BMLs also predict total knee replacement over periods of up to 4 years [49, 50, 73-75]. In some studies, BMLs predicted knee replacements more strongly than other predictors assessed in the same cohort $[49,74]$. Although these studies were of small size, this suggests that BMLs are a more sensitive predictor of development or progression of knee OA than other factors. An advantage of using BMLs as a measurement of disease progression or response to therapy is that response to treatment can be observed in a shorter period of time. In trials using BMLs as an outcome, differences in BML size were seen over periods as short as 6 weeks when looking at a brace for knee patellofemoral OA [76•] and 12 months when looking at the discrimination between OA treatment [53].

In vivo evidence for a subchondral bone phenotype of $\mathrm{OA}$ includes data on persons treated with bone remodelling drugs including bisphosphonates and strontium ranelate. Laslett et al. reported reduction in pain and reduction in size of BMLs in people with knee pain and clinical knee OA over 6 months using

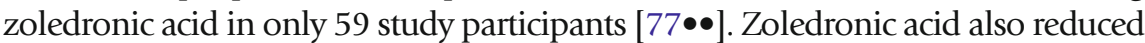
pain over 1 month in a similar pathology (Modic changes) in 40 people with low back pain, but there was no data regarding the effect of this drug on other structural changes in this cohort [78]. In a subset of the observational Osteoarthritis Initiative cohort, data showed improvements in pain for up to 3 years, and a trend toward reduction in JSN by year 4 in participants using any type of bisphosphonate [79]. In a phase III knee OA trial, strontium ranelate reduced both cartilage volume loss and BML and demonstrated an association between the decrease in BML score and the reduction in cartilage volume loss over 36 months [69••]. Other clinical trials evaluating drugs not directly related to bone remodelling revealed a strong association for the compounds tested between BML score change and cartilage volume loss in the same or related topographical sites [80] as well as the need for total knee replacement [49, 50, 53].

There is much less data for other anatomical sites. However, in a study on the hip, large BMLs were found to be longitudinal predictors of increased likelihood of high cartilage signal [81]. In the hand, BMLs were also shown to predict radiographic progression [82].

Therefore, targeting subchondral bone has allowed assessment of response to therapy for preventing disease progression using surrogate endpoints in relatively small numbers of participants over short periods of time. 
Effusion and/or synovitis are the hallmarks of joint inflammation. Such features can be measured using MRI without contrast agent quantitatively for the synovial fluid [83] and semi-quantitatively for the synovial membrane [84].

Data from the Osteoarthritis Initiative demonstrate that presence of either effusion or Hoffa synovitis predicts incident radiographic knee OA over 2 years [85]. Longitudinal observational data demonstrates that knee effusion/synovitis in the suprapatellar pouch predicts cartilage defects, BMLs and increased cartilage loss over 2.6 years, in a dose-response manner [86]. Interestingly, associations between synovial inflammation/effusion and cartilage alterations could be the 'chicken or the egg' dilemma in that, although synovial inflammation could lead to cartilage damage [87], the reverse is also possible [87, 88]. The presence of MRI-detected effusion or synovitis predicts knee replacement [75]. Data also showed that chondroitin sulphate, a symptomatic slow acting drug for osteoarthritis (SYSADOA) with DMOAD activity, at 6 months in patients concomitantly taking NSAIDs, significantly reduced the synovial membrane thickness (as assessed by MRI without contrast agent [84]) compared to the placebo group, in addition to decreasing cartilage volume loss [53].

MRI-detected synovitis has also been used as a measure of disease progression in hand OA, with the presence of moderate/severe synovitis predicting radiographic progression (JSN) over 5 years, along with BMLs, bone damage and osteophytes [82]. Trials are starting to use effusion/synovitis as a treatment target. In a trial using low-dose oral prednisolone for painful hand OA, MRIassessed effusion-synovitis was associated with pain cross-sectionally, but not hand pain as assessed by VAS; however, effusions did not change over time with treatment or predict response to treatment [89].

Meniscal alterations are associated with increased risk of developing radiographic knee OA in middle-aged and elderly individuals, with meniscal tears increasing the odds by 5.7 in one study [90] and meniscal damage increasing the hazard ratio of radiographic OA in 2 years by 1.81 in another [85]. Meniscal extrusion independently predicts knee cartilage volume loss at about 2 years [54, 91], as do meniscal tears [92]. Both meniscal extrusion [75] and tears [49] predict knee replacement surgery [49]. Meniscal tears also demonstrate effect modification through changes in weight, with increase in weight associated with increased cartilage loss over 2 years in those with meniscal tears; this effect was absent in those without meniscal tears [93•]. Importantly, co-localised knee meniscal extrusion/damage and BML further increased the risk of cartilage loss [94•, 95].

Although the presence of meniscal extrusion has been shown to be associated with greater cartilage volume loss and to be a strong marker of OA progression $[27,28]$, to our knowledge, there has been no clinical trial using MRI investigating the effect of meniscal extrusion as a primary outcome on response to drug treatment. However, post hoc analyses of studies exploring the effect of potential DMOADs argue in favour of an MRI-based diagnosis of meniscal extrusion in clinical practice, which would help physicians identify knee OA patients who are more likely to benefit from such treatment, namely 
those with mild-moderate OA with meniscal extrusion or those with more symptomatic disease but without meniscal extrusion $[54,94 \bullet, 96]$.

\section{Ultrasound}

Ultrasound is not yet widely used for assessing OA disease progression. Ultrasound is operator dependent, which increases measurement error if more than one operator images joints in the same research study. However, ultrasound is less expensive than radiography and MRI and could be used in clinical practice as both a diagnostic and explanatory tool for clinical symptoms. Ultrasound is more appropriate for superficial joints such as the hand rather than joints located deeper within the body including the hip.

\section{Ultrasound-detected inflammation}

In a study detecting inflammation using ultrasound, inflammation was associated with radiographic features, with the presence of power Doppler signals (but not presence of grey scale (GS) synovitis or effusions) and associated with new radiographic central erosions in the hand over 4 years [97]. In another study, grade of ultrasound-detected inflammation (GS synovitis, power Doppler signals) showed a positive dose-response association with progression of KL grade in hand OA over 5 years [98].

\section{Effusion}

Ultrasound-detected knee effusion predicts knee joint replacement [33]. In terms of treatment, the presence of ultrasound-detected knee effusions has been used to select people who might respond well to oral methotrexate, which demonstrated efficacy in an open-label trial, albeit without a control group [99]. Overall, although there is some evidence of use of ultrasound indices to assess disease progression in OA; mostly for effusion, there are very few studies for associations between abnormal ultrasound findings and other OA features or disease progression.

\section{Conclusions}

Progression of OA has historically been measured using radiographs; however, this method has major limitations including its inability to assess other important joint structures such as synovium, menisci and BMLs, in addition to being insensitive to change. MRI is increasingly used to assess OA disease progression and as a marker for diagnosis of the disease. This method has increased and will continue to increase our knowledge of the predictors of progression, which will in turn be important for optimal treatment allocation and clinical investigations of DMOAD therapies. Not all patients will progress symptomatically, radiologically, or to joint replacement, and treatments need to be focused on those most in need. Methodologies in regard to quantitative measures of MRI structures are now well used and provide a better understanding of factors associated with disease markers and progression. In turn, the joint structures 
as evaluated by MRI could help us to understand the extent of response to treatment as well as being used as targets in clinical investigations of new therapies. In this context, fully automated quantitative MRI systems for cartilage volume [100•], BML [101], osteophytes [102] and synovial fluid [83] were recently developed. A primary advantage of such fully automated systems for knee tissue structure is the possibility of intensive and autonomous computation, enabling images from large-scale studies to be analysed in a shorter time and, very importantly, compared to manual or semi-automated methods, increased stability of the reading, and hence increased accuracy of results.

Ultrasound requires more studies on joint features other than effusion/ synovitis in order to be a choice of method for OA patient diagnosis and disease progression.

\section{Compliance with Ethical Standards}

\section{Conflicts of Interest}

LLL and FMC declare that they have no conflicts of interest. JPP reports he is shareholder for ArthroLab Inc. GJ reports grants and honoraria from and advisory board membership with Roche; consultancy with Servier; advisory board membership with Sanofi and Jannsen; grants and speaker fees from Abbvie; a travel grant and speaker fees from BMS; speaker fees from Mundipharma; and grants from Ardea, Auxilium, and Inventiv, outside the submitted work. JMP reports that she is a shareholder for ArthroLab Inc.

\section{Human and Animal Rights and Informed Consent}

This article does not contain any studies/experiments with human or animal subjects performed by any of the authors not previously published.

\section{References and Recommended Reading}

Papers of particular interest, published recently, have been highlighted as:

- Of importance

- Of major importance

1. Lane NE, Brandt K, Hawker G, et al. OARSI-FDA initiative: defining the disease state of osteoarthritis. Osteoarthritis Cartilage. 2011;19:478-82.

2. Creamer P, Hochberg MC. Osteoarthritis. Lancet. 1997;350:503-8.

3. Loeser RF, Goldring SR, Scanzello CR, et al. Osteoarthritis: a disease of the joint as an organ. Arthritis Rheum. 2012;64:1697-707.

4. Walsh DA, Chapman V. Bisphosphonates for osteoarthritis. Arthritis Res Ther. 2011;13:128.

5. Martel-Pelletier J, Pelletier JP. Is osteoarthritis a disease involving only cartilage or other articular tissues? Eklem Hastalik Cerrahisi Joint Dis Relat Surg. 2010;21:2-14.

6. Quasnichka HL, Anderson-MacKenzie JM, Tarlton JF, et al. Cruciate ligament laxity and femoral intercondylar notch narrowing in early-stage knee osteoarthritis. Arthritis Rheum. 2005;52:3100-9.

7. Anderson-MacKenzie JM, Billingham ME, Bailey AJ. Collagen remodeling in the anterior cruciate ligament associated with developing spontaneous murine osteoarthritis. Biochem Biophys Res Commun. 1999;258:763-7.

8. Pelletier J-P, Kapoor M, Martel-Pelletier J. Animal models of osteoarthritis. In: Hochberg M, Silman AJ, Smolen JS, Weinblatt ME, Wiesman MH, editors. Rheumatology. 6th ed. Philadelphia: Elsevier; 2014. p. 1454-61.

9. Bijlsma JW, Berenbaum F, Lafeber FP. Osteoarthritis: an update with relevance for clinical practice. Lancet. 2011;377:2115-26. 
10. Lievense AM, Bierma-Zeinstra SM, Verhagen AP, et al. Prognostic factors of progress of hip osteoarthritis: a systematic review. Arthritis Rheum. 2002;47:556-62.

11. Roemer FW, Guermazi A, Niu J, et al. Prevalence of magnetic resonance imaging-defined atrophic and hypertrophic phenotypes of knee osteoarthritis in a population-based cohort. Arthritis Rheum. 2012;64:429-37.

12. Kellgren JH, Lawrence JS. Radiological assessment of osteo-arthrosis. Ann Rheum Dis. 1957;16:494-502.

13. Altman RD, Gold GE. Atlas of individual radiographic features in osteoarthritis, revised. Osteoarthritis Cartilage. 2007;15:A1-56.

14. Jones G, Ding C, Scott F, et al. Early radiographic osteoarthritis is associated with substantial changes in cartilage volume and tibial bone surface area in both males and females. Osteoarthritis Cartilage. 2004; 12:169-74.

15. Bruyere O, Honore A, Rovati LC, et al. Radiologic features poorly predict clinical outcomes in knee osteoarthritis. Scand J Rheumatol. 2002;31:13-6.

16. Bedson J, Croft PR. The discordance between clinical and radiographic knee osteoarthritis: a systematic search and summary of the literature. BMC Musculoskelet Disord. 2008;9:116.

17. Hannan MT, Felson DT, Pincus T. Analysis of the discordance between radiographic changes and knee pain in osteoarthritis of the knee. J Rheumatol. 2000;27:1513-7.

18. Michel A, Kohlmann T, Raspe H. The association between clinical findings on physical examination and self-reported severity in back pain. Results of a population-based study. Spine. 1997;22:296-303.

19. Dahaghin S, Bierma-Zeinstra SM, Reijman M, et al. Prevalence and determinants of one month hand pain and hand related disability in the elderly (Rotterdam study). Ann Rheum Dis. 2005;64:99-104.

20. Bruyere $\mathrm{O}$, Genant $\mathrm{H}$, Kothari $\mathrm{M}$, et al. Longitudinal study of magnetic resonance imaging and standard Xrays to assess disease progression in osteoarthritis. Osteoarthritis Cartilage. 2007;15:98-103.

21. Duryea J, Neumann G, Niu J, et al. Comparison of radiographic joint space width with magnetic resonance imaging cartilage morphometry: analysis of longitudinal data from the Osteoarthritis Initiative. Arthritis Care Res (Hoboken). 2010;62:932-7.

22. Hall J, Laslett LL, Martel-Pelletier J, et al. Change in knee structure and change in tibiofemoral joint space width: a five year longitudinal population-based study. BMC Musculoskelet Disord. 2016;17:25.

23. Cicuttini F, Hankin J, Jones G, et al. Comparison of conventional standing knee radiographs and magnetic resonance imaging in assessing progression of tibiofemoral joint osteoarthritis. Osteoarthritis Cartilage. 2005;13:722-7.

24. Raynauld JP, Martel-Pelletier J, Berthiaume MJ, et al. Long term evaluation of disease progression through the quantitative magnetic resonance imaging of symptomatic knee osteoarthritis patients: correlation with clinical symptoms and radiographic changes. Arthritis Res Ther. 2006;8:R21.

25. Hunter DJ, Zhang YQ, Tu X, et al. Change in joint space width: hyaline articular cartilage loss or alteration in meniscus? Arthritis Rheum. 2006;54:2488-95.

26. Hunter DJ, Zhang YQ, Niu JB, et al. The association of meniscal pathologic changes with cartilage loss in symptomatic knee osteoarthritis. Arthritis Rheum. 2006;54:795-801.

27. Berthiaume MJ, Raynauld JP, Martel-Pelletier J, et al. Meniscal tear and extrusion are strongly associated with progression of symptomatic knee osteoarthritis as assessed by quantitative magnetic resonance imaging. Ann Rheum Dis. 2005;64:556-63.

28. Pelletier JP, Raynauld JP, Berthiaume MJ, et al. Risk factors associated with the loss of cartilage volume on weight-bearing areas in knee osteoarthritis patients assessed by quantitative magnetic resonance imaging: a longitudinal study. Arthritis Res Ther. 2007;9:R74.

29. Conaghan PG, Hunter DJ, Maillefert JF, et al. Summary and recommendations of the OARSI FDA osteoarthritis Assessment of Structural Change Working Group. Osteoarthritis Cartilage. 2011;19:606-10.

30. Reichmann WM, Maillefert JF, Hunter DJ, et al. Responsiveness to change and reliability of measurement of radiographic joint space width in osteoarthritis of the knee: a systematic review. Osteoarthritis Cartilage. 2011;19:550-6.

31. Hunter DJ, Zhang W, Conaghan PG, et al. Responsiveness and reliability of MRI in knee osteoarthritis: a meta-analysis of published evidence. Osteoarthritis Cartilage. 2011;19:589-605.

32. Aitken D, Ding C, Pelletier JP, et al. Responsiveness of magnetic resonance imaging-derived measures over 2.7 years. J Rheumatol. 2014;41:2060-7.

33. Conaghan PG, D'Agostino MA, Le Bars M, et al. Clinical and ultrasonographic predictors of joint replacement for knee osteoarthritis: results from a large, 3year, prospective EULAR study. Ann Rheum Dis. 2010;69:644-7.

34. Bruyere O, Richy F, Reginster JY. Three year joint space narrowing predicts long term incidence of knee surgery in patients with osteoarthritis: an eight year prospective follow up study. Ann Rheum Dis. 2005;64:1727-30.

35. Cicuttini FM, Jones G, Forbes A, et al. Rate of cartilage loss at two years predicts subsequent total knee arthroplasty: a prospective study. Ann Rheum Dis. 2004;63:1124-7.

36. Hawker GA, Guan J, Croxford R, et al. A prospective population-based study of the predictors of undergoing total joint arthroplasty. Arthritis Rheum. 2006;54:3212-20.

37. Verbruggen G, Veys EM. Numerical scoring systems for the anatomic evolution of osteoarthritis of the finger joints. Arthritis Rheum. 1996;39:308-20.

38. Verbruggen G, Wittoek R, Vander Cruyssen B, et al. Morbid anatomy of 'erosive osteoarthritis' of the 
interphalangeal finger joints: an optimised scoring system to monitor disease progression in affected joints. Ann Rheum Dis. 2010;69:862-7.

39. Visser AW, Boyesen P, Haugen IK, et al. Radiographic scoring methods in hand osteoarthritis-a systematic literature search and descriptive review. Osteoarthritis Cartilage. 2014;22:1710-23.

40. Maheu E, Cadet C, Gueneugues S, et al. Reproducibility and sensitivity to change of four scoring methods for the radiological assessment of osteoarthritis of the hand. Ann Rheum Dis. 2007;66:464-9.

41. Bijsterbosch J, Haugen IK, Malines C, et al. Reliability, sensitivity to change and feasibility of three radiographic scoring methods for hand osteoarthritis. Ann Rheum Dis. 2011;70:1465-7.

42. Verbruggen G, Wittoek R, Vander Cruyssen B, et al. Tumour necrosis factor blockade for the treatment of erosive osteoarthritis of the interphalangeal finger joints: a double blind, randomised trial on structure modification. Ann Rheum Dis. 2012;71:891-8.

43. Kwok WY, Bijsterbosch J, Malm SH, et al. Validity of joint space width measurements in hand osteoarthritis. Osteoarthritis Cartilage. 2011;19:1349-55.

44. Hunter DJ, Arden N, Conaghan PG, et al. Definition of osteoarthritis on MRI: results of a Delphi exercise. Osteoarthritis Cartilage. 2011;19:963-9.

45. Haugen IK, Østergaard M, Eshed I, et al. Iterative development and reliability of the OMERACT Hand Osteoarthritis MRI Scoring System. J Rheumatol. 2014;41:386-91.

46. Pelletier JP, Cooper C, Peterfy $\mathrm{C}$, et al. What is the predictive value of MRI for the occurrence of knee replacement surgery in knee osteoarthritis? Ann Rheum Dis. 2013;72:1594-604.

47. Hunter DJ, Zhang W, Conaghan PG, et al. Systematic review of the concurrent and predictive validity of MRI biomarkers in OA. Osteoarthritis Cartilage. 2011;19:557-88.

48. Wildi LM, Martel-Pelletier J, Abram F, et al. Assessment of cartilage changes over time in knee osteoarthritis disease-modifying osteoarthritis drug trials using semiquantitative and quantitative methods: pros and cons. Arthritis Care Res. 2013;65:686-94.

This is the first head to head study demonstrating in an RCT that quantitative MRI is more sensitive than semi-quantitative methods in knee OA patient treatment response.

49. Raynauld JP, Martel-Pelletier J, Haraoui B, et al. Risk factors predictive of joint replacement in a 2-year multicentre clinical trial in knee osteoarthritis using MRI: results from over 6 years of observation. Ann Rheum Dis. 2011;70:1382-8.

50. Raynauld JP, Martel-Pelletier J, Dorais M, et al. Total knee replacement as a knee osteoarthritis outcome: predictors derived from a 4-year long-term observation following a randomized clinical trial using chondroitin sulfate. Cartilage. 2013;4:219-26.

51. Raynauld JP, Martel-Pelletier J, Berthiaume MJ, et al. Correlation between bone lesion changes and cartilage volume loss in patients with osteoarthritis of the knee as assessed by quantitative magnetic resonance imaging over a 24-month period. Ann Rheum Dis. 2008;67:683-8.

52. Eckstein F, Kwoh CK, Boudreau RM, et al. Quantitative MRI measures of cartilage predict knee replacement: a case-control study from the Osteoarthritis Initiative. Ann Rheum Dis. 2013;72:707-14.

53. Wildi LM, Raynauld JP, Martel-Pelletier J, et al. Chondroitin sulphate reduces both cartilage volume loss and bone marrow lesions in knee osteoarthritis patients starting as early as 6 months after initiation of therapy: a randomised, double-blind, placebo-controlled pilot study using MRI. Ann Rheum Dis. 2011;70:982-9.

54. Raynauld JP, Martel-Pelletier J, Bias P, et al. Protective effects of licofelone, a 5-lipoxygenase and cyclo-oxygenase inhibitor, versus naproxen on cartilage loss in knee osteoarthritis: a first multicentre clinical trial using quantitative MRI. Ann Rheum Dis. 2009;68:938-47.

55. Khan H, Aitken D, Ding C, et al. Natural history and clinical significance of cartilage defects over 10 years in a mid-life cohort[abstr]. Osteoarthritis Cartilage. 2015;23:A55-A6.

56. Wluka AE, Ding C, Jones $\mathrm{G}$, et al. The clinical correlates of articular cartilage defects in symptomatic knee osteoarthritis: a prospective study. Rheumatology (Oxford). 2005;44:1311-6.

57. Gold GE, Hargreaves BA, Stevens KJ, et al. Advanced magnetic resonance imaging of articular cartilage. Orthop Clin North Am. 2006;37:331-47. vi.

58. Stannus OP, Jiang D, Cicuttini F, et al. Cartilage signal intensity on T1-weighted MRI: association with risk factors and measures of knee osteoarthritis. Clin Rheumatol. 2014;33:359-68.

59. Burstein D, Velyvis J, Scott KT, et al. Protocol issues for delayed Gd(DTPA)(2-)-enhanced MRI (dGEMRIC) for clinical evaluation of articular cartilage. Magn Reson Med. 2001;45:36-41.

60. Crema MD, Hunter DJ, Burstein D, et al. Association of changes in delayed gadolinium-enhanced MRI of cartilage (dGEMRIC) with changes in cartilage thickness in the medial tibiofemoral compartment of the knee: a 2 year follow-up study using 3.0 T MRI. Ann Rheum Dis. 2014;73:1935-41.

61. Anandacoomarasamy A, Leibman S, Smith G, et al. Weight loss in obese people has structure-modifying effects on medial but not on lateral knee articular cartilage. Ann Rheum Dis. 2012;71:26-32.

62. Roos EM, Dahlberg L. Positive effects of moderate exercise on glycosaminoglycan content in knee cartilage: a four-month, randomized, controlled trial in patients at risk of osteoarthritis. Arthritis Rheum. 2005;52:3507-14.

63. Dunn TC, Lu Y, Jin $\mathrm{H}$, et al. T2 relaxation time of cartilage at MR imaging: comparison with severity of knee osteoarthritis. Radiology. 2004;232:592-8.

64. Jungmann PM, Kraus MS, Nardo L, et al. T(2) relaxation time measurements are limited in monitoring progression, once advanced cartilage defects at the knee 
occur: longitudinal data from the osteoarthritis initiative. J Magn Reson Imaging. 2013;38:1415-24.

65. Felson DT, McLaughlin S, Goggins J, et al. Bone marrow edema and its relation to progression of knee osteoarthritis. Ann Intern Med. 2003;139:330-6.

66. de Lange BJ, Ioan-Facsinay A, Bijsterbosch J, et al. The patellofemoral and femorotibial joints are related based on patterns of MRI features and their association with radiologic progression[abstr]. Osteoarthritis Cartilage. 2014;22:S254-S5.

67. Dore D, Martens A, Quinn S, et al. Bone marrow lesions predict site-specific cartilage defect development and volume loss: a prospective study in older adults. Arthritis Res Ther. 2010;12:R222.

68. Roemer FW, Guermazi A, Javaid MK, et al. Change in MRI-detected subchondral bone marrow lesions is associated with cartilage loss: the MOST Study. A longitudinal multicentre study of knee osteoarthritis. Ann Rheum Dis. 2009;68:1461-5.

69.•• Pelletier JP, Roubille C, Raynauld JP, et al. Diseasemodifying effect of strontium ranelate in a subset of patients from the phase III knee osteoarthritis study SEKOIA using quantitative MRI: reduction in bone marrow lesions protects against cartilage loss. Ann Rheum Dis. 2015;74:422-9.

This is the first study showing that a bone remodeling agent, strontium ranelate, significantly reduced, in addition to bone marrow lesions, the cartilage volume loss in knee OA patients.

70. Wluka AE, Hanna F, Davies-Tuck M, et al. Bone marrow lesions predict increase in knee cartilage defects and loss of cartilage volume in middle-aged women without knee pain over 2 years. Ann Rheum Dis. 2009;68:850-5.

71. Davies-Tuck ML, Wluka AE, Forbes A, et al. Development of bone marrow lesions is associated with adverse effects on knee cartilage while resolution is associated with improvement-a potential target for prevention of knee osteoarthritis: a longitudinal study. Arthritis Res Ther. 2010;12:R10.

72. Teichtahl AJ, Wluka AE, Wang Y, et al. Effect of longterm vigorous physical activity on healthy adult knee cartilage. Med Sci Sports Exerc. 2012;44:985-92.

73. Tanamas SK, Wluka AE, Pelletier JP, et al. Bone marrow lesions in people with knee osteoarthritis predict progression of disease and joint replacement: a longitudinal study. Rheumatology (Oxford). 2010;49:2413-9.

74. Scher C, Craig J, Nelson F. Bone marrow edema in the knee in osteoarthrosis and association with total knee arthroplasty within a three-year follow-up. Skeletal Radiol. 2008;37:609-17.

75. Roemer FW, Kwoh CK, Hannon MJ, et al. Can structural joint damage measured with MR imaging be used to predict knee replacement in the following year? Radiology. 2015;274:810-20.

76. Callaghan MJ, Parkes MJ, Hutchinson CE, et al. A randomized trial of a brace for patellofemoral osteoarthritis targeting knee pain and bone marrow lesions. Ann Rheum Dis. 2015;74:1164-70.
PF BMLs can change in as little as 6 weeks, and respond to PF knee bracing.

77.• Laslett L, Dore D, Quinn S, et al. Zoledronic acid reduces knee pain and bone marrow lesions over 1 year: a randomised controlled trial. Ann Rheum Dis. 2012;71:1322-8.

This is the first study to demonstrate that in human knee OA patients, a bisphosphonate, zoledronic acid, can modify bone marrow lesions as well as reducing pain.

78. Koivisto K, Kyllonen E, Haapea M, et al. Efficacy of zoledronic acid for chronic low back pain associated with Modic changes in magnetic resonance imaging. BMC Musculoskelet Disord. 2014;15:64.

79. Laslett LL, Kingsbury SR, Hensor EM, et al. Effect of bisphosphonate use in patients with symptomatic and radiographic knee osteoarthritis: data from the Osteoarthritis Initiative. Ann Rheum Dis. 2014;73:824-30.

80. Wildi LM, Raynauld JP, Martel-Pelletier J, et al. Relationship between bone marrow lesions, cartilage loss and pain in knee osteoarthritis: results from a randomised controlled clinical trial using MRI. Ann Rheum Dis. 2010;69:2118-24.

81. Ahedi H, Aitken D, Blizzard L, et al. A populationbased study of the association between hip bone marrow lesions, high cartilage signal, and hip and knee pain. Clin Rheumatol. 2014;33:369-76.

82. Haugen IK, Slatkowsky-Christensen B, Boyesen P, et al. MRI findings predict radiographic progression and development of erosions in hand osteoarthritis[epub before print]. Ann Rheum Dis 2016;75:117-23.

83. Li W, Abram F, Pelletier JP, et al. Fully automated system for the quantification of human osteoarthritic knee joint effusion volume using magnetic resonance imaging. Arthritis Res Ther. 2010;12:R173.

84. Pelletier JP, Raynauld JP, Abram F, et al. A new noninvasive method to assess synovitis severity in relation to symptoms and cartilage volume loss in knee osteoarthritis patients using MRI. Osteoarthritis Cartilage. 2008;16 Suppl 3:S8-13.

85. Roemer FW, Kwoh CK, Hannon MJ, et al. What comes first? Multitissue involvement leading to radiographic osteoarthritis: magnetic resonance imaging-based trajectory analysis over four years in the Osteoarthritis Initiative. Arthritis Rheum. 2015;67:2085-96.

86. Wang X, Blizzard L, Halliday A, et al. Association between MRI-detected knee joint regional effusionsynovitis and structural changes in older adults: a cohort study. Ann Rheum Dis 2016;75:519-25.

87. Ayral X, Pickering EH, Woodworth TG, et al. Synovitis: a potential predictive factor of structural progression of medial tibiofemoral knee osteoarthritis-results of a 1 year longitudinal arthroscopic study in 422 patients. Osteoarthritis Cartilage. 2005;13:361-7.

88. Sellam J, Berenbaum F. The role of synovitis in pathophysiology and clinical symptoms of osteoarthritis. Nat Rev Rheumatol. 2010;6:625-35.

89. Wenham CY, Hensor EM, Grainger AJ, et al. A randomized, double-blind, placebo-controlled trial of low-dose oral prednisolone for treating painful hand 
osteoarthritis. Rheumatology (Oxford).

2012;51:2286-94.

90. Englund M, Guermazi A, Roemer FW, et al. Meniscal tear in knees without surgery and the development of radiographic osteoarthritis among middle-aged and elderly persons: the Multicenter Osteoarthritis Study. Arthritis Rheum. 2009;60:831-9.

91. Sharma L, Eckstein F, Song J, et al. Relationship of meniscal damage, meniscal extrusion, malalignment, and joint laxity to subsequent cartilage loss in osteoarthritic knees. Arthritis Rheum. 2008;58:1716-26.

92. Roemer FW, Zhang Y, Niu J, et al. Tibiofemoral joint osteoarthritis: risk factors for MR-depicted fast cartilage loss over a 30-month period in the multicenter osteoarthritis study. Radiology. 2009;252:772-80.

93. Teichtahl AJ, Wluka AE, Wang Y, et al. The longitudinal relationship between changes in body weight and changes in medial tibial cartilage, and pain among community-based adults with and without meniscal tears. Ann Rheum Dis. 2014;73:1652-8.

Weight loss slows down cartilage loss and improves pain in people with meniscal tears.

94. $\quad$ Roubille C, Martel-Pelletier J, Raynauld JP, et al. Meniscal extrusion promotes knee osteoarthritis structural progression: protective effect of strontium ranelate treatment in a phase III clinical trial. Arthritis Res Ther. 2015;17:82.

Presence of meniscal extrusion can modify the response to treatment in knee OA patients.

95. Roemer FW, Felson DT, Wang K, et al. Co-localisation of non-cartilaginous articular pathology increases risk of cartilage loss in the tibiofemoral joint-the MOST study. Ann Rheum Dis. 2013;72:942-8.
96. Roubille C, Martel-Pelletier J, Abram F, et al. Impact of disease treatments on the progression of knee osteoarthritis structural changes related to meniscal extrusion: data from the OAI progression cohort. Semin Arthritis Rheum. 2015;45:257-67.

97. Mancarella L, Addimanda O, Pelotti P, et al. Ultrasound detected inflammation is associated with the development of new bone erosions in hand osteoarthritis: a longitudinal study over 3.9 years. Osteoarthritis Cartilage. 2015;23:1925-32.

98. Mathiessen A, Slatkowsky-Christensen B, Kvien $\mathrm{TK}$, et al. Ultrasound-detected inflammation predicts radiographic progression in hand osteoarthritis after 5 years[epub before print]. Ann Rheum Dis 2015. doi:10.1136/annrheumdis-2015207241

99. Wenham CY, Grainger AJ, Hensor EM, et al. Methotrexate for pain relief in knee osteoarthritis: an openlabel study. Rheumatology (Oxford). 2013;52:888-92.

100. Dodin P, Pelletier JP, Martel-Pelletier J, et al. Automatic human knee cartilage segmentation from 3D magnetic resonance images. IEEE Trans Biomed Eng 2010;57.

These are the first MRI systems that assess in a fully quantitative automated fashion the cartilage volume [100] and bone marrow lesions [101] in knee OA patients.

101. Dodin P, Abram F, Pelletier J-P, et al. A fully automated system for quantification of knee bone marrow lesions using MRI and the osteoarthritis initiative cohort. J Biomed Graph Comput. 2013;3:51-65.

102. Dodin P, Martel-Pelletier J, Pelletier JP, et al. A fully automated human knee 3D MRI bone segmentation using the ray casting technique. Med Biol Eng Comput. 2011;49:1413-24. 